Proceedings

\title{
Inheritance is a Surjection: Description and Consequence ${ }^{+}$
}

\author{
Paul Ballonoff \\ Ballonoff Consulting, 9307 Kings Charter Drive, Mechanicsville, VA 23116, USA; Paul@Ballonoff.net; \\ Tel.: +1-703-780-1761 \\ + Presented at the 5th International Electronic Conference on Entropy and Its Applications, 18-30 November \\ 2019; Available online: https://ecea-5.sciforum.net/.
}

Published: 17 November 2019

\begin{abstract}
Consider an evolutionary process. In genetic inheritance and in human cultural systems, each new offspring is assigned to be produced by a specific pair of the previous population. This form of mathematical arrangement is called a surjection. We have thus briefly described the mechanics of genetics - physical mechanics describes the possible forms of loci, and normal genetic statistics describe the results as viability of offspring in actual use. However, we have also described much of the mechanics of mathematical anthropology. Understanding that what we know as inheritance is the result of finding surjections and their consequences is useful in understanding, and perhaps predicting, biological-as well as human-evolution.
\end{abstract}

Keywords: surjection; biological evolution; mathematical anthropology; predicting biological evolution; predicting cultural evolution; mathematical groups

\section{Description of Study}

The basic mathematical description from finding inheritance is a surjection is summarized in Section 2, below. By concentrating on the notion of "surjection," we are forced to observe, from the mathematical theorems in Section 2, that two things must occur simultaneously: the offspring population must all be unique individuals, and the parental pairs must be using identical or isomorphic means of creating the offspring. Our normal discussion of phylogenetics imply that the parents are using the "same" genetic means (thus, that their treatments are identical or isomorphic), but the results of Section 2 shows that we must prove that.

Thus, Section 3 provides a detailed description that all offspring are unique, both for humans and other offspring. In Section 3, we also present the Mendelian arrays of results of breeding relationships and their traditional statistics. Nothing in seeing that inheritance is a surjection changes what the methods of Section 3 detect. We must also recognize that all parental "couples" act under identical or isomorphic methods allowed, and all are acting under identical (isomorphic) methods allowed by specific mathematical groups. The proof is in the articles of Section 4 .

For population genetics, to count the results using possible different alleles at each locus, we must count the total number of surviving offspring from each parental pair. In physical mechanics, the creation and the intervals of reproduction are potentially observable within normal human perception; usually, they occur in very short periods. Thus, in population genetics, counting the number of offspring from each pair of "parents" is the actual number of offspring surviving from each pair that reproduce.

In culture theory, the existing literature shows that mathematical groups occur as the means of describing the mechanism of each culture's rules of kinship and marriage. However, human cultural systems may require decades of time for one generation to be replaced by another. Each human 
culture has its own culturally determined means of assigning offspring to parental pairs. Depending on the culturally specific rules, each parental pair's offspring may include their own genetic offspring but may also follow culturally designated devices such as adoption. Thus, for human cultures, the assigned offspring may include individuals who may have no genetic relation to the "parents."

However, when we recognize that the human means of describing kinship and marriage is also a surjection, we see from Section 2 that we can apply the Stirling Number of the Second Kind (SNSK). Cultural rules allow that offspring are not necessarily biologically related to their assigned parents. Use of the SNSK statistics, however, permits us to find the expected population statistics without assuming they are biologically related. Using SNSK thus allows us to interpret the past and make forecasts of the future in association with the culturally specific rules of marriage, which traditional demographic analysis does not treat. This result is discussed in Sections 5 and 6 below. The results in Section 6 also shows us that recognition of surjection allows us to treat issues that have not been treated by other methods; we thus give specific examples, and how the analysis gives new and unique results. Our discussion in Sections 5 and 6 is a bit longer because the topic has not been broadly discussed, although the underlying principles, including examples, have been in published articles since 1982.

In Section 7, we discuss more recent issues, based on thoughts of predicting future biological and human evolution, as has been proposed in recent literature. The fact that future evolution is governed by a surjection also means that both the present condition and any future evolution must be described molecules determined by a limited set of mathematical groups. In other parts of physics, when results are based on mathematical groups, the future may also be formed into classes determined by those groups. We ask here if such result also follows for population genetics. The author suspects that this is likely the case, but here, we simply propose the question. Section 8 is a brief summary. A list of our basic definitions follows Section 8 .

\section{Foundation Discussion}

In genetics, biological descendants are the actual biological descendants of the identified parental pair. In human systems, descendants may be the actual biological descendants of the parental pair but may also reflect other cultural assignments, such as by (non-inherited) devices such as adoption. In both cases, we have a mapping from each offspring to a particular set of parents. The well know mathematical name for this relation is surjection. Theorems on how inferences are made for surjections include reference [1] p. 178, [2-4]. Look especially at reference [1] p. 178 and reference [4] for the author's "Twelvefold Way" (pages 69-79).

The theorems cited in references [1-4] show that finding that a relation is a surjection also means that the newly created offspring individuals are each "unique" and the entities to which offspring are assigned by their parental organisms are identical or isomorphic in how the parents act. Neither of those notions are in our concepts of the subjects here. We do not assume that surjection causes these results, but if we find out that they are not true, it means our assessment that the processes form a surjection is wrong. This we must first demonstrate if "uniqueness" and "identical or isomorphic forms of reproduction" are correct descriptions.

\section{Uniqueness of Offspring}

When we say that the offspring of an evolutionary process are unique, we mean that there is at least one (but perhaps many) differences between each of those offspring. Radzevilavicius and Blackstone's [5] very detailed review of the evolution of individuality of offspring populations shows that all individuals, of all species, created by evolution are also in some form unique; the reader should see that article for its many details. Thus, the authors of reference [5] conclude "since [our review] ... only relies on universal principles of life's evolution, this framework can potentially be applied to all life, no matter what its chemistry, and wherever it is found" (p. 1630).

The mathematics that describes how biological evolution works began in the late 19th century, representing the frequency or relative frequencies of alternative alleles at specific loci. If we define a 
single species, there are common sets of DNA/RNA that define that single species, but there may also be recombination of the alleles that can act at each loci $[5,6]$ made a similar observation. These variants may create features in specific offspring that are not determined by the common genes in all of that species. Thus despite isomorphism in the defining characteristics of a species (isomorphism in following the DNA/RNA genetic codes that define that specific species), the uniqueness of individuals includes some differences, which may be differences in specific DNA/RNA alleles, differences in recombination of DNA/RNA in their individual coding sequence, and from other genetically coded individual differences.

The first such description was by Mendel [7], followed in the early 20th century by Bernstein [8,9], Fischer [10], Haldane [11-20], Wright [21], Nei [22], Crow and Kimura [23], and others. There are useful overall comparisons of those methods including references [24-30] and others. Though each of those authors used somewhat different mathematical representations to determine the allele frequencies in specific cases, all showed that devices such as those of Mendel allow many different forms of inheritance. There are many possible locus sites in each organism, and in nearly all organisms, there may be diverse alleles at some or many sites; therefore, each individual may have hundreds, thousands, or indeed many multiple thousands of diverse sites per organism. Thus, individual uniqueness is not difficult to understand. While isomorphism of certain alleles at certain sites may define a specific species, the distinct frequencies in some or many other loci sites of individuals are one of the many reasons why unique individual types of each species exist.

\section{Identical Means of Reproduction}

We must also show that each of the genetic parental pairs must use identical or isomorphic means of reproduction. This has been shown since the basic mechanics for construction of DNA and RNA molecules are also structured by the group-theoretical results of physical mechanics. For the mechanisms of DNA, see for example the articles of Petoukhov [31-35], Petoukhov and CastroChaves [36], Petoukhov and Hu [37], Petoukhov, Petoukhova and Svirin [38], Hainen [39], Rakocevic [40], and others, who have demonstrated that the DNA devices used for genetic reproduction are described by mathematical groups by physical mechanics. For RNA, see similar work, also using similar groups of 64 codons that have been described by Khrennikov and Kozyrev, Rakocevic, and by A. Dragovich and B. Dragovich [41-46]. A simple basic description of the genetic mechanisms represented is found, for example, in reference [38], using descriptions of the 64 codons of DNA with the arrangements of all members in accordance with the binary-oppositional signs of the bases $\mathrm{A}, \mathrm{C}$, $G$, and T.

While the specific objects of the genes (DNA and/or RNA) in each act creating new offspring are distinct objects, the fact that all must satisfy specific group-theoretical forms means that the isomorphisms of those group theoretical forms (required by how alleles may form under the allowed physical mechanics), which means that all organisms are formed using the specific sets of mathematical groups, thus acting under isomorphic rules of combination. They thus are following what being part of surjections implies they must be doing.

\section{Kinship}

Ballonoff $[47,48]$ recently reviewed the literature of mathematical anthropological description, in relation to the ability of a system to survive. The earliest paper for mathematical description of kinship is from 1882: [49] modeled strings of symbols to represent semantic concepts, such as "MBD" (mother's brother's daughter) for an instance of part of the English term "first cousin." However, in 1945, ethnographer Ruheman [50] created the first description of kinship that identified objects that are clearly mathematical groups.

Ruheman denoted each cultural system by placing names of kinship relations onto finite sets of offspring in the culture's language, used diagrams to show how a terminology relates those sets of labels onto representative minimal set of parents, and assumed each system had distinct generations. She used labels A and B for moiety names. Descents of a male of A are also called A; descendants of 
a male of $B$ are also called $B$; the top layer is one generation of two sets of moieties, with two marriages, which reproduces in one generation another set of A and B moieties. Her illustrations use "=" for a "marriage" between two individuals, two sets of parallel but opposite arrows between individuals as "brothers and/or sisters," and placed objects into columns labelled $\mathrm{m}$ and $\mathrm{f}$ for males and females. The arrows or = signs on each end of each line connect to the persons on the other end of each line.

This system thus has at least two sets of marriages in each generation; each A always marries a $B$. In each generation, each generation minimal structure has two marriages. That minimal group has an order; let the minimal group size order $=\mathrm{s}$. The structural number of a rule is equal to the size of the minimal group representing that rule. Thus, for Figure 1, the structural number of that rule is $s=$ 2.

\begin{tabular}{|c|c|c|c|c|c|c|c|c|}
\hline & $f_{1}$ & & $m_{1}$ & & $f_{2}$ & & $m_{2}$ & \\
\hline$\vec{I}$ & $\begin{array}{l}\text { A } \\
\text { B }\end{array}$ & $=$ & $\begin{array}{l}\text { B } \\
\text { B }\end{array}$ & $\rightleftarrows$ & $\begin{array}{l}\text { B } \\
\text { A }\end{array}$ & $=$ & $\begin{array}{l}A \\
\text { A }\end{array}$ & $\leftarrow$ \\
\hline
\end{tabular}

Figure 1. Example from Ruheman's study of Australian kinship.

To create a diagram like Figure 1, reference [50] said: “... The system must be self-contained and consistent; must provide a sufficient number of descent lines (a) to enable every member to select a spouse from his own generation; (b) to allow space for every distinct relationship term; and no [kinship] term may appear more than twice ... in any one generation. ... The system must be able to reproduce itself after a number of generations...." (p. 543).

Reference [50] said that by using mathematical representations, "... It is independent of any special principles, which might at best be controversial, and proceeds simply from a systematic arrangement of the recorded facts; ... It enables the dominating features of even a very complex system of kinship terms to be brought into full view in a single picture; ... It makes possible the comparison and classification of widely divergent kinship systems, and provides a useful check on the completeness of our information" (p. 576). Ruheman's methods do not therefore discuss many of the issues often treated in non-mathematical analysis of cultural systems. Avoiding nonmathematical descriptions is one of her purposes.

The mathematical groups for each minimal set of kinship terms described by Ruheman (or Weil as cited in the next paragraph) is also now called the "minimal structure" [47] of that system of kinship description. It is also described $[47,48]$ as the "history" of that system since it describes how possible parental pairs of that culture may be created. The minimal structure is not a literal description of how all marriages work, though in some cases some empirical relations can also look like the Ruheman minimal descriptions in some ethnographies. The minimal kinship diagrams also look like the illustrations of regular genetic systems of inbreeding shown by Wright [21]. Just as in cultural analysis, the ability to show a minimal representation does not mean that all implementations of an experiment to do inbreeding must look exactly like the minimal form. But representations are very useful. Petoukhov [51] demonstrated that there are similar mathematics behind literary texts (thus including kinship in human languages) and long genetic texts. When analyzing human Dravidian kinship categories, Vaz [52] showed that mathematical representation of DNA/RNA were isomorphic to the mathematical groups that describe parts of the human Dravidian kinship categories.

While Ruheman created the notion, the most referenced "first" published text using group mathematics for kinship was by Andre Weil [53], in an appendix to Claude Levi-Strauss' 1947 The Elementary Structures of Kinship [54]. Weil's initiative was then expanded by [55-58]. Use of the related Cayley groups was described in reference [59].

One apparently difficulty of doing logical and algebraic manipulations on kinship data is that mathematical groups require an associative operation that creates the mathematical group on 
elements of the language. But human languages are not associative. In English, compare "Tom shows" to "shows Tom." Thus, the first work showing that kinship rules are actually non-associative algebras, but still consistent with the mathematics cited here, was reference [60]. A non-associative algebra can have an associative sub-set (the kinship rules for example), which are allowed by reference [60] and required by reference [48]. Reference [61] is the first paper which derives mathematical kinship groups entirely from purely mathematical assumptions. [62,63] elaborated many group-theoretical examples of kinship systems. Reference [64] reviewed Weil's original work and its subsequent history. Reference [65] extended Weil's work by using semigroups and homomorphisms among semigroups, as well as groups. Other extensions include references [66-71].

In 2018, a series of eight articles greatly expanded the depth of analysis of kinship [72-79]; readers should see those articles also for their added citations. Many of those papers also seeking to relate kinship groupings directly to other concepts of cultural order; [60] sought the same results. Thus, Read [73] (p. 54) cited the necessity of mathematical groups in kinship descriptions: "Because the core structure for classificatory terminologies has inverses and not just reciprocity between ascending and descending kin terms, the core structure is a group; that is, an algebra with an (associative) binary product, an identity element, and an inverse for each element in the algebra." Read is describing the empirical facts that make Ruheman's concepts necessary.

Finally, while this author does not advocate that all analysis use mathematical representations for all problems of human kinship (nor that they solve all relationship issues), Harris [61] at page 86, has considered this. His conclusion is,

This paper represents relations among individuals as mathematical relations ... and defines a relation "alter". ... "alter" is shows to be an equivalence relation, and the equivalence classes generated and the "categories of equivalent people" mentioned above ... can be pairs to form a "clan"... or to form a marriage type ... Moreover, our assumptions restricting allowable relations are used to prove as theorems the assumptions of previous authors restricting allowable permutation groups.

Thus, while this paper does not advocate that all authors use mathematical representations for all analysis, we believe that using the descriptions of human kinship terms using their minimal mathematical groups, is a valid basis for the analysis used here.

\section{Culture and Population Measurements}

Summarizing Section 5, knowing the order of a culture's minimal group, and using the fact that inheritance is a surjection, allows us to predict present and future populations statistics for that culture. In Section 6, we discuss how to do that, using the percentage of the population at time $t$ using a history $\alpha$ with structural number $s$, and the fact that for each $s$ there is are specific values $n_{s}$ (average family size) and $p_{s}$ (percentage of reproducing married females). We can then forecast demographic measures demography $n(t)$ and $p(t)$ found as weighted average of the percentage of society with structural number $\mathrm{s}$, added over the entire society at each $\mathrm{t}$. We define this in more depth below, and give actual examples.

Each culture assigns offspring individuals to a specified parental pair. For the "reproduction" assigned to each parental pair, there may be actual mating between them, but there may also be other means of the culture to assign offspring to that pair. These include "cheating" - sometimes known but often not known to other members of the population, assignment of other non-genetic offspring by adoption, and other means of assigning offspring to each parental pair. References [1-4] show that surjection means that the possible average numbers of offspring to parental pairs can be done by the Stirling Number of the Second Kind (SNSK), which we implement using Equations (1) through (4) below. While ethnographic studies are usually done from a few months to several years, observations over several decades are needed since the results are in "generations," and each generation typically takes 20-30 or so years. None the less, our empirical examples summarized below give results closely matching what the results from analysis of the potential structural numbers shows us.

Many things affect population size; this article is not an attempt to identify nor describe most of those. The demographic idea of the Leslie matrix [80,81] produces a growth rate and stable age 
distribution as eigenvalues and eigenvectors of a matrix of age-structured mortality and fertility or fecundity rates. This reflects the approach of Lotka [82] that the "normal" demographic discussion of the growth of populations is analogous to "capital" growth in economics. Neither the Leslie matrix nor the Lotka models treats the cultural system, and thus do not treat consequences on population change due to changes in the cultural system (that is, due to changes in the history).

In the illustration below, the formulas that determine the objects described by $\mathrm{D}$ are results from demographic processes. Definitions of the objects in the illustration are found in our Definitions section at end of this text. The surjection requirement of using SNSK as cited in Part 2 allows us to predict how the demographics - measures of of $n(t)$ and $p(t)-$ of a population may change, as the histories change. To explore this, reference [83] defined the following:

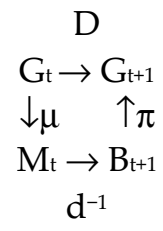

Assignments of offspring and parental pairs are, in each case, what are required by the specific rules of genetics or cultural assignment applied. We next show how to use the surjections of cultural theory to derive population estimate suing Equations (1) to (4) to understand the illustration. We follow by summarizing examples from previously published articles.

Let $\mathrm{s}$ be the structural number of history $\alpha$. Let $\mathrm{n}_{\mathrm{s}}=$ average family size of a system with structural number $\mathrm{s}$, and define the proportion of (socially ascribed) reproducing females as $\mathrm{p}_{\mathrm{s}}$. Let $p_{s}=2 / n_{s}$. The specific values of $p_{s}$, and $n_{s}$ by structural number $s$ are listed in reference [3] in their appendix 2 and further cited in $[47,84,85]$. Note that $n_{s}$ is always $\geq 2$, so $n^{*} p=2$, or

$$
1 / 2\left(n_{s}^{*} p_{s}\right)=1
$$

Since we can set $\mathrm{e}^{\mathrm{r}(\mathrm{t})}=1$, then we can also derive a general "equity growth" equation for any system with structural number s:

$$
\mathrm{e}^{\mathrm{r}(\mathrm{t})}=1 / 2 \mathrm{n}^{*} \mathrm{p}
$$

We call this exponential form $\mathrm{r}(\mathrm{t})$ "equity growth" to distinguish it from the "capital growth" idea per Lotka. But as Lotka also used the notation " $r(t)$ " for "capital growth" we here instead call Lotka's growth rate $R(t)$ to distinguish it from our own $r(t)$; in Equation (4) below we derive an estimate of capital growth $R(t)$ from the equity growth $r(t)$.

When we use the values $n_{s}$, $p_{s}$ given a history of structural number s, see reference [3] in their appendix 2. Then if we have just one structural number, since $\mathrm{n}_{\mathrm{s}} \mathrm{p}_{\mathrm{s}}=2$, then Equation (2) results in $\mathrm{r}(\mathrm{t})$ $=0$. When $r(t)=0$, it shows that the values of $n_{s}$ and $p_{s}$ allow a culture with history $\alpha$ and structural number $s$ to have neither growth nor decline. As structural number $s$ increases (above $s=3$ ), then from reference [3] in their appendix 2] and $[47,84,85]$ for a system with only one cultural rule (one history) values of $n_{s}$ increase and $p_{s}$ decrease, to maintain cultural stability with zero change $r(t)=0$. Another way to state this same result is that if the cultural systems gets "more complex" (as the structural number $\mathrm{s}$ increases) then the average family size $\mathrm{n}_{\mathrm{s}}$ per household also increases - that is similar to the notion that as general systems become more complex, their size must increase to maintain the same level of reliability - which here is when $\mathrm{r}(\mathrm{t})=0$.

Now, let the population at time $t$ use some set of histories $\alpha, \ldots, \beta$. Then the proportion of each generation population $\mathrm{Gt}_{\mathrm{t}}$ using history $\alpha$ is $\mathrm{v}_{\alpha \mathrm{t}}$, the proportion of $\mathrm{Gt}_{\mathrm{t}}$ using history $\beta$ is $\mathrm{v}_{\beta}$, etc. We require that $0 \leq \mathrm{v}_{\alpha \mathrm{t}}, \ldots, \mathrm{v}_{\beta t} \leq 1$ and $\Sigma_{\mathrm{v}_{\alpha \mathrm{t}}}=1$ over all histories allowed in time period $t$. Then the average family size $n(t)=\Sigma v_{\alpha} t_{s}$ over all histories allowed at time $t$; the average proportion of marries females allowed to fertilize is $\mathrm{p}(\mathrm{t})=\Sigma \mathrm{v}_{\alpha \mathrm{t}} \mathrm{p}_{\mathrm{s}}$ over all histories allowed at time $t$. We thus get an equation that allows prediction at time $t$ of the time-dependent average population measures for $n(t), p(t)$ and $r(t)$ as the percentages of histories $\alpha, \ldots, \beta$ change:

$$
e^{r(t)}=1 / 2 n(t)^{*} p(t)
$$


Thus, in summary, $\mathrm{r}(\mathrm{t})=0$ whenever all rules have the same structural number (or all have only $\mathrm{s}=2$ or 3$)$. But $\mathrm{r}(\mathrm{t}) \neq 0$ whenever there is a change in cultural dynamics by allowing rules with more than one structural number, and at least one of structural number $>3$; all such changes cause population growth (or decline). This is well known by anthropologists: as cultures change, population statistics can also change. It is however predicted by the mathematics of cultural theory. We need to use SNSK, as revealed by the mathematics of surjection, to find this.

To relate $r(t)$ to the demographic notion $R(t)$ we applied the mathematics originally developed for analysis of financial leverage (see reference [86] Chapters 3 and 4; reference [84], especially its Equation (17); and reference [85]), which result in

$$
R(t)=2 p(t) r(t) /[p(t) 2+2 r(t)]
$$

Equation (4) is also called the amplification equation since it increases the usually much smaller equity growth value of $r(t)$ into a larger capital growth value $R(t)$. It can also be called "amplification" of local survivability, or, by analogy to borrowing of capital in the world of finance, "leverage." The reader can look at references $[47,48,84,85,87,88]$ for more details and citations of numerous examples.

We now summarize some results below. One of the useful results of finding cultural rules of kinship and marriage are surjections is that the theorems of Section 2 allows us to apply the statistics of the statistic SNSK. Most of the "standard statistics" taught in academic statistics courses are in fact adaptations of the distributions of volumes of identical gas particles, distributed into specific (and distinct) parts of real space. But surjection means we have unique offspring, being assigned to parents who use identical or isomorphic means of process. The assumptions of the surjection are thus the opposite of what is required compared to standard academic statistics. However, the statistics of SNSK are exactly what is required: unique objects (as individuals in the offspring population), and similar means of treatment by the parents.

This means that by using that we have a surjection, the SNSK allows us to make important predictions of what may exist in real ethnographies, which can also be verified. Thus [84] used the changes in the structural numbers as listed in the table in reference [3] in their appendix [2] to predict the changes in Western European population statistics from about 1000 AD to about 1950 AD. Generally, early in that period we had relatively higher structural numbers in Western Europe, since for example, the Catholic Church had higher degrees of prohibition of relatives; thus, higher structural numbers, and thus higher values of $\mathrm{ns}$. By the middle of the 20th century, there were much higher percentages of the population following more "Protestant" rules, which in general had lower

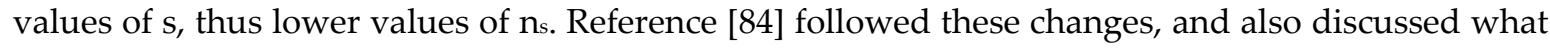
may have happened when the black-plague took place-it may be that when that happened, there were also lower densities of marriageable people in local populations, but they needed a high $\mathrm{n}_{\mathrm{s}}$. The ability of the population to create that $n_{s}$ what thus not physically present. The Protestant changes took place in that period and also led to a new marriage rule lowering the $\mathrm{n}_{\mathrm{s}}$ for the newer Protestant populations. We do not claim here that our statistics tell use exactly what changes, or that what we call "Protestant" were the only choices. However, reference [48] may include that the possible changes among the available sets of histories at time $t$, might also limit what choices are available, and thus may limit what alternate forms of histories might be possible. The reader might also look at examples such as references $[87,88]$, which discuss not only at the values of $n_{s}$ and $p_{s}$ but also how the distributions of choices (the mechanics of the application of the $\mathrm{d}^{-1}$ devices in our diagram) move parts of the certain other cultural practices when a village might be subdividing. There are thus many things that need to be better understood in ethnographies, which can be better explained when the use of proper statistics (here, using SNSK) is allowed.

Reference [48], as noted above, also discusses that some combinations of possible histories might be unable to combine with one another, so not all means of potential cultural evolution might be possible. There are probably less than 20 distinct forms of how kinship systems may be created (though there may be thousands of cultures with distinct languages in which they are expressed); and most of those systems have structural numbers at or well under $s=16$. Thus, from reference [48], not all combinations of possible cultures create a lattice whose top element creates a lattice whose 
top-group order is perhaps well above $s=16$. Empirically therefore there may be no such systems. Another way to state this is that there may be sets of possible histories, not all of which can be allowed to find each other from within that particular set of histories. That result may create changes among histories in which some ranges of values of $n(t)$ and of $p(t)$-and thus their consequences in predicting $r(t)$ (and thus from Equation (4), $R(t)$ ), might not be allowed. The homotopy of these systems may therefore tell us a lot on which kinds of cultural systems might evolve into each other, and which, if any, might not evolve.

Many ethnographic studies discuss each cultural system as if there is only one history present in that population. In many ethnographic papers, therefore their discussion does not talk of "probabilities" that a culture is in some specific history; they assume a specific history, so no probabilities are needed. Applications of the same history at each instance of time also may imply that there is no change in demographics of the population: if the same structural number repeats, then the predictions of $n(t)$ and $p(t)$ do not change; a specific history has some structural number $s$, so the forecasts remain at $n(t)=n_{s}$ and $p(t)=p_{s}$; see also reference [47]. However, when probabilities are needed (i.e., that more than one history be present in a society), then in reference [48], the probabilities use Pauli coefficients; this is used in part because complex Hilbert spaces also simplify following specific paths.

The forms of mathematics found in Equation (1) through (4) also yield non-commutative forecasts. While some of our equations look like similar forms in quantum analysis, our results are not equivalent. One version of quantum mechanics [89-92] uses a "consistent histories" approach, in which the same history is consistently followed in each generation of a descent structure. This approach defines a descent map (called "descent" in quantum theory as well), which, like the descent map of genetics or cultural theory, is a partial order. In physics, the physical object in each instance of $t+1$ is a copy of the same object as in time $t$; only its momentum or location may have changed. Changing whether we measure first momentum or first location causes the problem of uncertainly in physics, despite the assumed consistency. But in cultural theory the objects at $t+1$ are descendants of the objects at $\mathrm{t}-$ they are distinct objects, not the same objects as assumed in physical theory. The authors of reference [48] found that when objects in culture theory are non-commutative, our equations forecast $r(t) \neq 0$ and thus also that generation growth (or decline) occurs. But the differences when finding results in cultural theory are directly computable. Thus, while Equations (1) through (4) are based on non-commutative cultural theory, and we get significant changes in values of $\mathrm{e}(\mathrm{t})$, $\mathrm{n}(\mathrm{t})$ and $\mathrm{p}(\mathrm{t})$, but we do not find uncertainly.

In the previous three paragraphs, we do not discuss similar details of how biological evolution may also occur or be limited by the mechanics of the mathematical groups required. However, somewhat similar problems also occur in population genetics. For example, some sequences of associations of certain alleles, might limit whether certain additional things can be added to that code. We assume that such details are better understood in normal treatment of genetics, hence we do not need to discuss then here; while these ideas for forming cultural systems are less often discussed.

\section{Forecasting Evolution}

The evolutionary biologist Wilson published his most current book, Genesis [93], in which the title apparently intends to forecast the next stage in evolution of life. It is apparent even from a sympathetic reviewer [94] that Wilson reaches no such conclusion. However, Wilson does what an evolutionary biologist specializing in social insects might do: he uses the long history of evolution of social insects to "model" what may happen next with the much shorter and later span of evolution of humans.

In our own discussion in Sections 4 and 6 we also discuss the possibility of forecasting. Can we use the notion of surjection to assist prediction in biological systems? In 1940, Pauling [95] used the mathematics of the chemical bond for description of the molecules used in evolution. Petoukhov [96] p. 26 says that Pauling's "presentation form is based on symmetric matrices ... Symmetrical matrices are related with the theory of resonance of L. Pauling ..." Section 4 of this paper tells us that any configuration that follows the constraints of Section 4 can also create symmetrical matrices (in fact, 
mathematical groups) that may cause evolution in their direction, so long as they follow the group requirements in Section 4. The proof of prediction that would simply be to demonstrate it has occurred, which it has (see also citations of Section 4). To the knowledge of this author, using symmetrical configurations to describe biological evolution was first described by Schroeck [97] (pp. 223-253) in bats. Schroeck is not describing either the loci or the alleles of evolution, but he is demonstrating the results. Reference [97] showed that bats identified other objects in flight by computations inside the bat, and when the speeds of object found by the bat became fast enough, by using quantum mechanical devices inside the bat to identify and follow the objects tracked. As the mechanisms of the other flying animals became fast enough, the limits (and result) of their locational devices was that "Detailed analysis ... shows that bats perform at the quantum limit of uncertainty relations ..." Schroeck then continues by proposing that "we observe that joint time/frequency spectrograms are being used as a standard analysis procedure in studying biological and social systems. We list the 'pictures' of bird songs in many popular bird identification manuals, as well as the identification of vocal expressions of stump-tailed macaques ... as examples" [97] (pp. 252-253). In fact, what Schroeck has stated is that the biological results are determined by the requirements of the physics. There is actually nothing unique in this, except it shows that "simply" the mechanics allowed by relations allowed by Section 4 have that result. (Continuing this discussion see also our citations below on Kauffman discussing communications among species.)

Returning to Wilsons notion, reference [95] says Wilson might better have compared the evolution of social insects to the evolution of human communications from understanding kinship (which is the opposite of what Wilson asserts). Read [96] shows that the (verbal!) notions of kinship analysis derive deeply in descriptions of human society. Dinosaurs, including modern birds, began many millions of years ago to use verbal communication with each other, which is also a primary feature of human evolution and human society. Indeed, if humans did not have verbal communication, how could we write the sections of this paper describing human ability to describe human society using kinship terminologies-which are part of human communication systems-and to then discuss the role of those using mathematical groups to understand the demography of human societies? But we have also found from the example described by Schroeck, that biological evolution is based on physical mechanical devices. In much of physical theory, forecasting future conditions are in large part due to their symmetry, given their mathematical groups. But also Read [98] at page 54 , cited the necessity of mathematical groups in kinship descriptions: “... Because the core structure for classificatory terminologies has inverses and not just reciprocity between ascending and descending kin terms, the core structure is a group; that is, an algebra with an (associative) binary product, an identity element, and an inverse for each element in the algebra."

So, let us restate the issue here: do the possible alleles that may be formed under mechanics allowed by our Section 4 citations, necessarily cause specific forms that define future evolution? That is not how we normally state definitions of potential evolution; at best, it places the roles of the computations of Part 3 in demonstrating the paths of particular results; but it is what Section 4 can create that determines the eventual results of evolution. Consider the results Hirschleifer [99]. Hirshleifer was an economist who showed that the logic of economic evolution (in effect, classical physical dynamics, as used in the computations of the Section 3 predictions) could allow biology to favor "sacrificial" individuals - "altruistic evolution" was thus found to be favored by ordinary classical mechanics devices. Indeed, much of our current understanding of how Darwinian theory explains human culture and how human creativity using mental processes causes social evolution, are fundamentally due to Hirshleifer [99]; see also [100].

Thus, biological evolution, as summarized above by Schroeck, also allows us to believe that human logical abilities (a result of biological evolution) can thus also cause human "social" evolution. Methods of how communication can occur [101] in biological evolution are very diverse; for example, in [101], Chapter 12 includes tweaks as animals move, which are very different from human verbal communications [101] in Chapters 13 and 14. Once some biological entity has communication capability, it is only a matter for time before some individual(s) of that species can use them to create languages, including mathematics (see Ezhkova $[102,103])$. This speculation is also proven since such 
things are already widely present - certainly in humans; and recent work shows birds [104] and other citations verify Schroeck's thoughts; other species may have at least parts of it. Kauffman [105,106] argues that evolution should also look for means of communication among species, says that "simple and complex systems can exhibit power full self-organization" but that "no body of thought incorporates self-organization into the weave of evolutionary theory" [105] p. vii; see also Mayr and Provine [107]. Section 4, however, was formed by understanding that we have a surjection; since surjection also implies the mathematics of group theory (Section 4); therefore, understanding surjection directly incorporates "self-organization" by use of mathematical groups.

Human logic (also a product of biological evolution) can already forecast results of much of mathematics of physics. The human brain (a product of biological evolution) can forecast future versions of many domesticated crops and animals; indeed so have other species that have "tended" crops. Then why can't humans forecast (indeed create) their own cultural and biological evolution? Humans themselves of course are part of evolution (see also reference [108]).

\section{Conclusions}

Physical mechanics (Section 4) allows us to describe the objects on which evolution can take place, while genetic statistics (Section 3) describes "only" the means of demonstrating empirical evolutionary effects in actual use. We need to understand both forms of prediction, and to understand how they relate to each other. However, evolution may only occur only in specific directions implied by what Section 4 devices can create and allow.

The foundation question is thus, given the things that Section 4 allows, do those necessarily cause the results of evolution? Can the mechanical foundations of biological evolution (Section 4) can lead us (humans!) to forecast how future biological, and cultural, evolution might occur? Can we (humans) predict them? The answers might well be "yes."

\section{Definitions}

generation-a defined limited but continuous duration of time, called $t$ members-a non-empty set of "individuals" at time of some species alive at time $t$ parents-a pair of members of the same generation who are assigned as creating new individuals at $t$ offspring-a set of individuals who are created in generation $t+1$ from the parents in $t$ evolutionary process-a process that creates, or changes what may be created, by means of defined interactions between two individuals of the same generation

genetics-an evolutionary process in which the parents are creating new individuals by means of transmission of genes:

-the position in the genome where there are or can be variants is a locus.

-the alternative forms of DNA at the position of a locus are alleles.

mathematical anthropology-a mathematical representation of a cultural evolutionary process in which the parents are identified by cultural rules and to which offspring are assigned.

surjection-a collection of two sets of non-empty objects $\mathrm{P}$ and $\mathrm{O}$, where $\mathrm{P} \cap \mathrm{O}=0$, and where each member of $\mathrm{P}$ has at least one member of $\mathrm{O}$ linked to it. (Note therefore that the members of $\mathrm{P}$ can be called "parents" and the members of $\mathrm{O}$ can be called "offspring".)

(mathematical) group-a non-empty set $\mathrm{G}$ with an operation + such that for each $\mathrm{g}$ in $\mathrm{G}$ :

-an object $\mathrm{o}$ in $\mathrm{G}$ such that for every $\mathrm{g}$ in $\mathrm{G}, \mathrm{g}+\mathrm{o}=\mathrm{o}+\mathrm{g}=\mathrm{g}$;

-an object in $\mathrm{G}$ called identity or I;

-for each $\mathrm{g}$ in $\mathrm{G}$ is an member $\mathrm{g}^{-1}$ in $\mathrm{G}$ such that for each $\mathrm{g}, \mathrm{g}+\mathrm{g}^{-1}=\mathrm{g}^{-1}+\mathrm{g}=\mathrm{I}$;

-for any pair of objects $g$, $h$ in $G, g+h$ and $h+g$ are members of $G$, and $g+h=h+g$.

\section{describing or transmitting cultural information:}

-are used to represent a particular cultural rule, also called a history. -the proportion of each generation population Gt using history is $\mathbf{v} \mathbf{t}$. 
-each cultural rule of marriage has a structural number $s$, which is set by the size of the minimal group representing that rule (page 4).

-let $s$ be the structural number of history.

-let $n_{s}=$ average family size (average number of offspring to a set of parents in time period $t$ ) of a system with structural number s.

-Define the proportion of (socially ascribed) reproducing females as $p_{s .}$ Let $p_{s}=2 / n_{s}$.

-The specific values of $\mathrm{p}_{\mathrm{s}}$, and $\mathrm{n}_{\mathrm{s}}$ are presented in [3 appendix 2]

Part 6, Equations (1) through (4) show how to predict for each $t$ :

-the value of the expected surviving average family size $n(t)$;

-average percent of assumed reproducing females $p(t)$;

-average expected change in population growth $\mathrm{r}(\mathrm{t})$;

The demographics papers [80-82] also use a notation $\mathrm{r}(\mathrm{t})$; since we use that here for a different concept, we thus need a distinct representation for that demographic result. We thus use $R(t)$ for the demographic concept described in [80-82] as $r(t)$. The relation between $r(t)$ and $R(t)$ are given by Equation (4).

\section{Mathematical diagram}

\section{Let: Mean:}

D biological population operators of the population at the indexed time $t$,

Gt is the population size at time $t$

$\mathrm{M}_{t}$ the sets of married or biologically mating couples at time $\mathrm{t}$, with $\mathrm{M}_{\mathrm{t}} \subseteq \mathrm{Gt}$

$\mathrm{B}_{\mathrm{t}+1}$ a partition of $\mathrm{G}_{\mathrm{t}+1}$ into sets of offspring with the same parents, with $\mathrm{B}_{\mathrm{t}+1} \subseteq \mathrm{G}_{\mathrm{t}+1}$

$\mu \quad$ a surjection corresponding to assignments of $\mathrm{Mt}_{\mathrm{t}}$

$\pi$ a partition of $\mathrm{G}_{t+1}$ showing kin groups of a population within a generation $t+1$, as assigned by the genetic or cultural rules of marriage

$\mathrm{d}^{-1}$ a surjection corresponding to descent

d an injection corresponding to ancestor so that the $\mathrm{d}^{-1}$ surjection maps the progress of population change showing sets of descendants in generation $t+1$ onto the sets of their parents in generation $t$.

This gives us a diagram summarizing our mathematical representations:

$$
\begin{gathered}
\mathrm{Gt}_{\mathrm{t}} \rightarrow \mathrm{G}_{\mathrm{t}+1} \\
\downarrow_{\mu} \uparrow_{\pi} \\
\mathrm{M}_{\mathrm{t}} \rightarrow \mathrm{B}_{\mathrm{t}+1} \\
\mathrm{~d}^{-1}
\end{gathered}
$$

\section{References}

1. Grimaldi, R.P. Discrete and Combinatorial Mathematics; Addison Wesley: Reading, MA, USA, 1989.

2. Hildon, P.; Peterson, J.; Stiger, J. On Partitions, Surjections and Stirling Numbers; Bulletin of the Belgian Mathematical Society; Belgian Mathematical Society: Brussels, Belgium, 1994; Volume 1, pp. 713-735.

3. Schadach, D. A Classification of Mappings; Ballonoff, P., Ed.; Reprinted in Appendix 2; Mathematical Theory of Culture; Austrian Society for Cybernetic Studies Monograph No. 40: Vienna, Austria, 1987.

4. Stanley, R.P. Enumerative Combinatorics, 2nd ed.; Cambridge University Press: Cambridge, UK, 2012; Volume 1.

5. Radzevilavicius, A.L.; Blackstone, N.W. The evolution of individuality revisited. Biol. Rev. 2018, 93, 16201633.

6. Zheng, W.; Zhao, H.; Mancera, E.; Steinmetz, L.; Snyder, M. Genetic Analysis of Variation in Transcription Factor Binding in Yeast. Nature 2010, 464, 1187-1191.

7. Mendel, G. Experiments in Plant Hybridisation; Reprinted 1965 with Introduction; Harvard University Press: Boston, MA, USA, 1865.

8. Bernstein, S. Demonstration mathematique de la loi d'heredite de Mendel. Compt. Rend. Sci. Math. 1923, 177 Pt. 1, 528-531. 
9. Bernstein, S. Principe de stationarite et generalisations de la loi deMendel. Compt. Rend. Sci. Math. 1923, 177 Pt. 1, 581-584.

10. Fisher, R.A. The Genetical Theory of Natural Selection; Oxford University Press: Oxford, UK, 1930.

11. Haldane, J.B.S. A Mathematical Theory of Natural and Artificial Selection" (Part I). Trans. Camb. Philos. Soc. 1924, 23, 19-41.

12. Haldane, J.B.S. A Mathematical Theory of Natural and Artificial Selection" (Part II). Biol. Rev. Camb. Philos. Soc. 1924, 158-163.

13. Haldane, J.B.S. A Mathematical Theory of Natural and Artificial Selection" (Part III). Proc. Camb. Philos. Soc. 1926, 23, 363-372.

14. Haldane, J.B.S. A Mathematical Theory of Natural and Artificial Selection" (Part IV). Proc. Camb. Philos. Soc. 1926, 23, 607-615.

15. Haldane, J.B.S. A Mathematical Theory of Natural and Artificial Selection" (Part V). Proc. Camb. Philos. Soc. 1926, 23, 838-844.

16. Haldane, J.B.S. A Mathematical Theory of Natural and Artificial Selection" (Part VI). Proc. Camb. Philos. Soc. 1930, 26, 220-230.

17. Haldane, J.B.S. A Mathematical Theory of Natural and Artificial Selection" (Part VII). Proc. Camb. Philos. Soc. 1930, 27, 131-136.

18. Haldane, J.B.S. A Mathematical Theory of Natural and Artificial Selection" (Part VIII). Proc. Camb. Philos. Soc. 1930, 27, 137-142.

19. Haldane, J.B.S. A Mathematical Theory of Natural and Artificial Selection" (Part IX). Proc. Camb. Philos. Soc. 1931, 28, 244-248.

20. Haldane, J.B.S. The Causes of Evolution; Original 1942; Princeton University Press: Princeton, NJ, USA, 1990.

21. Wright, S. Evolution and the Genetics of Populations: Volume 2, The Theory of Gene Frequencies; University of Chicago Press: Chicago, IL, USA, 1969.

22. Nei, M. Molecular Evolutionary Genetics; Columbia University Press: New York, NY, USA, 1987.

23. Crow, J.F.; Kimura, M. An Introduction to Populations Genetics Theory; Harper and Row: New York, NY, USA, 1970.

24. Provine, W.B. The Origins of Theoretical Population Genetics; republished 2001; University of Chicago Press: Chicago, IL, USA, 1971.

25. Provine, W.B. Sewall Wright and Evolutionary Biology; University of Chicago Press: Chicago, IL, USA, 1986.

26. Lewontin, R.C. The Doctrine of DNA; Penguin Books: London, UK, 1992.

27. Ewens, W.J. Mathematical Population Genetics, I. Theoretical Introduction, 2nd ed.; Springer: New York, NY, USA, 2000.

28. Rice, S.H. Evolutionary Theory: Mathematical and Conceptual Foundations; Sinauer Associates: Sunderland, MA, USA, 2004.

29. Okasha, S. Evolution and the Levels of Selection; Oxford University Press: Oxford, UK, 2006.

30. Dronamaraju, K. Haldane, Mayr, and Beanbag Genetics; Oxford University Press: Oxford, UK, 2011.

31. Petoukhov, S.V. Matrix Genetics and Algebraic Properties of Multi-Level System of Genetic Alphabets. NeuroQuabtatology 2011, 9, 799-820.

32. Petoukhov, S.V. The degeneracy of the genetic code and Hadamard matrices. arXiv 2008, arXiv:0802.3366.

33. Petoukhov, S.V. Matrix genetics, part 1: Permutations of positions in triplets and symmetries of genetics matrices. Inst. Phy 2013, arXiv:0803.0888v6.

34. Petoukhov, S.V. Genetic coding and united-hypercomplex systems in the models of algebraic biology. Biosystems 2017, 158, 31-46.

35. Petoukhov, S.V. The Genetic Coding Systems and Unitary Matrices a Publication of the Institute of Machines Science; Russian Academy of Science: St. Petersburg, Russia, 2019.

36. Petoukhov, S.V.; Castro-Chavez, F. A Tetrahedral Representation of the Genetic Code Emphasizing Aspects of Symmetry. BioComplexity 2012, 2, 1-6.

37. Petoukhov, S.V.; Hu, Z.B. Generalized crystallography, the genetic system and biochemical esthetics. Struct. Chem. 2017, 28, 239-247.

38. Petoukhov, S.V.; Petoukhova, E.; Svirin, V. Genetic Alphabetic Alphabets, Unitary Matrixes and QuantumAlgorithmic Genetics; ToPME; IOP Publishing: Bristol, UK, 2019; pp. 1-5.

39. Hainen, P.C. An octonion models for physics. In Proceedings of the 4 th Conference on Emergence, Coherence, Hierarchy, and Organization (ECHO 4), Denmark, 2000. 
40. Rakocevic, M. Golden and Harmonic Mean in the Genetic Code; TABIX: Belgrade, Serbia, 2013.

41. Khrennikov, A.; Kozyrev, A.V. Replica procedures for probabilistic algorithms as a model of gene duplication. arXiv 2011, arXiv:1104.2893v1.

42. Khrennikov, A.; Kozyrev, A.V. Genetic code on the dyadic plane. arXiv 2007, arXiv:070107v3.

43. Khrennikov, A. Hyperbolic quantum mechanics. arXiv 2000, arXiv:0101002v1.

44. Khrennikov, A.; Kozyrev, A.V. p-Adic numbers in bioinformatics: From genetic code to PAM-Matrix. arXiv 2009, arXiv:0903.0137.

45. Dragovich, B.; Dragovich, A. A p-Adic Model of DNA Sequence and Genetic Code. arXiv 2006, arXiv:0607018v1.

46. Rakocevic, M.M. The Cipher of the Genetic Code. Biosystems 2018, 171, 31-47.

47. Ballonoff, P. Progress of Mathematical Cultural Theory. Math. Anthropol. Cult. Theory Lett. Rev. Mact 2018, 1-18.

48. Ballonoff, P. Paths of Cultural Systems. Entropy 2018, 20, 1-14.

49. Macfarlane, A. Analysis of Relationships of Consanguinity and Affinity. J. R. Anthropol. Soc. 1882, 12, 4663.

50. Ruheman, B. A method for analyzing classificatory relationship systems. Southwest. J. Anthropol. 1945, 1, 531-576.

51. Petoukhov, S.V. Structural Connections between Long Genetic and Literary Texts; A publication of the Institute of Machines Science; Russian Academy of Science: St. Petersburg, Russia, 2019.

52. Vaz, R.M. Relatives, Molecules, Particles. Math. Anthropol. Cult. Theory 2014, 7, 1-157.

53. Weil, A. 1947 Appendix to Part 1, pp. 221-229 to Claude Levi-Strauss, original 1947, cited here as 1969.

54. Claude Levi-Strauss 1947, current 1969. The Elementary Structures of Kinship; Beacon Press: Boston, MA, USA, 1969.

55. White, H. An Anatomy of Kinship; Prentice Hall: New Jersey, NJ, USA, 1963.

56. Liu, P.-H. Formal Analysis of Prescriptive Marriage System: The Murngin Case. Viii Congr. Anthropolo. Eth. Sci. Vol. Ethnol. 1968, 90-92.

57. Liu, P. Murngin: A mathematical solution, Institute of Ethnology. Acad. Sin. Nankang, Taipei, 1970.

58. Courrege, P. Un Modele mathematique des structures elementairs de parente. In L Homme; Ballonoff, P.A., Ed.; Genetics and Social Structure, Dowden, Hutchinson and Ross: Stroudsburgh Pennsylvania, PA, USA, 1974; Volume 5, pp. 248-290.

59. Gould, S.; Kronenfeld, D. New System for the Formal Analysis of Kinship; University Press of America: New York, NY, USA, 2000.

60. Greechie, R.; Martin, O. An Introduction to a Mathematical Approach to Kinship. In Genealogical Mathematics; Ballonoff', P., Ed.; Maison des Sciences de l'Homme: Paris, France, 1974; pp. 63-85.

61. Harris, T.R. From Relations to Groups: A Formal Treatment of the Underlying Logic of Some GroupTheoretical Models of Kinship. In Genealogical Mathematics; Ballonoff', P., Ed.; Maison des Sciences de l'Homme: Paris, France, 1974; pp. 85-109.

62. Gottscheiner, A. On some classes of kinship systems, I: Abelian systems. Math. Anthropol. Cult. Theory 2008, 2, 1-10.

63. Gottscheiner, A. On some classes of kinship systems, II: Non-Abelian systems. Math. Anthropol. Cult. Theory 2008, 2, 1-10.

64. Rauff, J.R. The algebra of marriage: An episode in applied group theory. J. Br. Soc. Hist. Math. 2016, 31, 230244.

65. Cargal, J. An Analysis of the Marriage Structure of the Murnguin Tribe of Australia. Behav. Sci. 1968, 23, 157-168.

66. Fat, F.E.T.S. Circulating connubium and transitive ranking: A second solution to Leachs problem. Bijdr. Tot De Taal Land-En Volkenkd. 1983, 139, 140-151.

67. Fat, F.E.T.S. Age Metrics and Twisted Cylinders: Predictions from a Structural Model. Am. Ethnol. 1983, 10, 585-604.

68. Fat, F.E.T.S. On the Formal Analysis of 'Dravidian', 'Iroquois', and 'Generational' Varieties as NearlyAssociative Combinations. In Transformations of Kinship; Fat, F.E.T.S., Godelier, M., Eds.; Smithsonian Institution Press: Washington, DC, USA; London, UK, 1998; pp. 59-93.

69. Fat, F.E.T.S. Representing Kinship: Simple Models of Elementary Structures. Thesis Leiden. 1990.

70. Fat, F.E.T.S. On Mathematics in Structural Theory. Curr. Anthropol. 1980, 21, 386-391. 
71. Fat, F.E.T.S.; Atkins, J.R.; Barnard, A.; Buchler, I.; De Meur, G.; Eyde, D.B.; Fischer, M.; Jorion, P.; Rustad, J.A. More Complex Formulae of Generalized Exchange [and Comments and Replies]. Curr. Anthropol. 1981, 22, 377-399.

72. Read, D. The Generative Logic of Crow-Omaha Terminologies: The Thonga-Ronga Kinship Terminology as a Case Study. Math. Anthropol. Cult. Theory 2018, 12, 1-37.

73. Read, D. Reply to Comments on The Generative Logic of Crow-Omaha Terminologies: The Thonga-Ronga Kinship Terminology as a Case Study. Math. Anthropol. Cult. Theory 2018, 12, 1-62.

74. Heady, P. Comment on D. Read 'Generative Crow-Omaha Terminologies'. Math. Anthropol. Cult. Theory 2018, 12, 1-8.

75. Fat, F.E.T.S. Comment on D. Read 'Generative Crow-Omaha Terminologies'. Math. Anthropol. Cult. Theory 2018, 12, 1-10.

76. Hamberger, K. Comment on D. Read 'Generative Crow-Omaha Terminologies'. Math. Anthropol. Cult. Theory 2018, 12, 1-9.

77. Heady, P. Comment on D. Read 'Generative Crow-Omaha Terminologies'. Math. Anthropol. Cult. Theory 2018, 12, 1-4.

78. Trautmann, T.R.; Peter, M. Whiteley Comment on D. Read 'Generative Crow-Omaha Terminologies'. Math. Anthropol. Cult. Theory 2018, 12, 1-12.

79. De Almeda, M.B. Comment on D. Read 'Generative Crow-Omaha Terminologies'. Math. Anthropol. Cult. Theory 2018, 12, 1-23.

80. Leslie, P.H. On the use of matrices in population mathematics. Biometrika 1945, 33, 183-212.

81. Leslie, P.H. Some further notes on the use of matrices in population mathematics. Biometrika 1945, 35, 213245.

82. Lotka, A. Relation Between Birth and Death Rates. Science 1907, 26, 435-438.

83. Duchamp, T.; Ballonoff, P. Mathematical Foundations of Social Anthropology; Ballonoff, P., Ed.; Mouton: Paris, France, 1975; Chapter 3.

84. Ballonoff, P. Mathematical Demography of Social Systems. In Progress in Cybernetics and Systems Research; Trappl, R., Ed.; Hemisphere Publishing Company: Washington, DC, USA, 1982; Volume X, pp. 101-112.

85. Ballonoff, P. Mathematical Demography of Social Systems II. In Cybernetics and Systems Research; Trappl, R., Ed.; North Holland Publishing Co: Amsterdam, The Netherlands, 1982; Volume XI, pp. 555-560.

86. Ballonoff, P. Mathematical Theory of Culture. In Austrian Society for Cybernetic Studies Monograph No. 40; World Scientific Publishing Company: Vienna, Austria, 1987.

87. Ballonoff, P. Theory of Lineage Organization. Am. Anthropol. 1983, 85, 70-91.

88. Ballonoff, P. Structural statistics: Models relating demography and social structure with applications to Apache and Hopi. Soc. Biol. 1973, 20, 421-426.

89. Isham, C.J. Quantum Logic and Decohering Histories. arXiv 1995, arXiv:9506028.

90. Isham, C.J. Lectures on Quantum Theory; Imperial College Press, London: 1995.

91. Sorkin, R.D. Space time and causal sets. In Proceedings of the Relativity and Gravitation, Proceedings of the Conf. Silarg VII, Coyoc, Mexico, December 1990.

92. Sorkin, R.D.; Rideout, D.P. A Classical Sequential Growth Dynamics for Causal Sets. Phys. Rev. 1999, 61, 024002.

93. Wilson, E.O. Genesis: The Deep Origin of Societies; Liveright Publishing: New York, NY, USA, 2019.

94. Denham, W. Sociality in E.O. Wilson's Genesis: Expanding the Past, Imagining the Future. Math. Anthropol. Cult. Theory 2019, 14, 1-37.

95. Pauling, L. The Nature of the Chemical Bond and the Structure of Molecules and Crystals: An Introduction to Modern Structural Chemistry, 2nd ed.; Oxford University Press: London, UK, 1960.

96. Petoukhov, P.; Petoukhov, S. Hyperbolic Numbers in Modeling Genetic. Phenom. Prepr. 2019, doi:10.20944/preprints201908.0284.v2.

97. Schroeck, F. Quantum Mechanics on Phase Space; Kluwer Academic Publishers: Dordrecht, The Netherlands, 1996.

98. Read, D. From past to present: The deep history of kinship. In Integrating Qualitative and Social Science Factors in Archaeological Modelling; Saqalli, M., Vander Linden, M., Eds.; Computational Social Sciences; Springer International Publishing: New York, NY, USA, 2019; Chapter 6; doi:10.1007/978-3-030-12723-7_6.

99. Hirshleifer, J. Economics from a Biological Viewpoint. J. Law Econ. 1977, 20, 1-52.

100. Hausken, K.: Jack Hirshleifer: A Nobel Prize left unbestowed. Eur. J. Political Econ. 2006, 22, 251-276. 
101. D'ettorre, P.; Hughes, D.P. (Eds.) Sociobiology of Communication; Oxford University Press: Oxford, UK, 2008.

102. Ezhkova, I. Self-organizing representations. Cybern. Syst. 2005, 36, 861-875.

103. Ezhkova, I. The Principles of Cognitive Relativity, Rationality and Clarity: Application to Cultural Theory. Cybern. Syst. 2004, 35, 229-257.

104. Pepperberg, I.M. The Alex Studies, Cognitive and Communicative Abilities of Grey Parrots; Harvard University Press: Cambridge, UK, 1999.

105. Hauffman, S. Reinventing the Sacred; Oxford University Press: Oxford, UK, 1993.

106. Hauffman, S. The Origins of Order: Self-Organization and Selection in Evolution; Oxford University Press: Oxford, UK, 2008.

107. Maye, M.; Provine, W. (Eds.) The Evolutionary Synthesis: Perspectives on the Unification of Biology; Harvard University Press: Cambridge, UK, 1980.

108. Ballonoff, P. On the Evolution of Self Awareness. In Cybernetics and Systems; Trapple, R., Ed.; Austrian Society for Cybernetic Studies: Vienna, Austria, 2000; Volume I, pp. 347-352.

(C) 2019 by the authors. Licensee MDPI, Basel, Switzerland. This article is an open access article distributed under the terms and conditions of the Creative Commons Attribution (CC BY) license (http://creativecommons.org/licenses/by/4.0/). 